L'exploration contemporaine de la biodiversité. Approche anthropologique de l'expédition Santo 2006

\title{
Elsa Faugère
}

\author{
(2) OpenEdition \\ Journals \\ Édition électronique \\ URL : http://journals.openedition.org/jso/5462 \\ DOI : $10.4000 /$ jso.5462 \\ ISSN : $1760-7256$ \\ Éditeur \\ Société des océanistes \\ Édition imprimée \\ Date de publication : 15 décembre 2008 \\ Pagination : 195-206 \\ ISBN : 978-2-85430-012-3 \\ ISSN : 0300-953x
}

Référence électronique

Elsa Faugère, « L'exploration contemporaine de la biodiversité. Approche anthropologique de l'expédition Santo 2006 », Journal de la Société des Océanistes [En ligne], 126-127 | Année 2008, mis en ligne le 15 décembre 2011, consulté le 19 juin 2020. URL : http://journals.openedition.org/jso/5462 ; DOI : https://doi.org/10.4000/jso.5462 


\title{
L'exploration contemporaine de la biodiversité. Approche anthropologique de l'expédition Santo 2006
}

par

\author{
Elsa FAUGÈRE*
}

\section{RÉSUMÉ}

Dans les années 1980, les scientifiques alertent les médias, les politiques et l'opinion publique internationale sur l'existence d'une grave crise affectant la diversité biologique, qu'ils appellent alors "biodiversité 》. Dès lors, des initiatives internationales vont se faire jour pour tenter d'apporter des réponses à cette crise. Ces réponses doivent d'abord surmonter le " handicap taxonomique ${ }^{\prime}$ "défini dans les conventions internationales comme un manque de connaissances taxonomiques sur la biodiversité aggravé par le nombre insuffisant de spécialistes susceptibles de le combler, limitant ainsi notre capacité à découvrir, à gérer et à utiliser la biodiversité. L'expédition Santo 2006, dont l'objectif était de faire un inventaire taxonomique aussi complet que possible des compartiments de la biodiversité de l'île de Santo au Vanuatu, peut être vue comme l'une de ces réponses. Elle inaugure en effet un nouveau type d'expédition scientifique, nouveau par la taille, l'échelle d'intervention, la pluridisciplinarité, la médiatisation, le respect des règles éthiques fixées par la Convention sur la diversité biologique, la privatisation d'une partie importante de son financement et l'importance donnée aux invertébrés, terrestres et marins, et aux espèces invasives, compartiments négligés de la biodiversité. Cet article interroge, d'une manière plus générale, la signification anthropologique de cette expédition scientifique contemporaine en explorant notamment la question des rapports, mar chands et non marchands, à la science et à la nature qu'elle a impliqués etlou révélés.

Mots-CLÉS: Vanuatu, Santo, biodiversité, expéditions, déficit taxonomique

\begin{abstract}
During the eighties, the scientists alerted the media, the politicians and the international public opinion on the crisis affecting the biological diversity, so called "biodiversity". Since then, different kinds of international initiatives have arisen in order to provide some answers to this crisis. These answers must overcome the "taxonomic impediment", that is the gap of our knowledge in the taxonomic system applying to biologically unexplored areas and the shortage of trained taxonomists and curators, both reasons having odd impacts on our ability to discover, to manage and to use the biological diversity. The Santo 2006 Expedition can be considered as one of these answers. It represents a new kind of scientific expedition, new by its size, its scale of study, its pluridisciplinarity, its mediatisation, the respect of ethical rules fixed by the Convention on Biological Diversity, the important part of private funding, and of invertebrates and invasive species, which constitute a neglected component of biodiversity. This paper questions the anthropological meaning of such a scientific expedition. It explores the question of the relationships, market-based and non market-based, to science and nature that the expedition included andlor revealed.
\end{abstract}

KeYwords: Vanuatu, Santo, biodiversity, expeditions, taxonomic impediment

1. NDLR. - Nous aurions préféré « déficit taxonomique » pour traduire taxonomic impediment.

* Ethnologue, Écodéveloppement INRA—Avignon, UR 0767, faugere@avignon.inra.fr 
Pendant la période coloniale, les expéditions scientifiques ont joué un rôle clé non seulement dans l'acquisition de nouveaux savoirs sur la diversité naturelle et culturelle - certains de ces savoirs ont conduit à des applications importantes (en termes agroalimentaires, pharmaceutiques, cosmétiques...) - mais aussi dans la construction des empires coloniaux occidentaux. Sciences, politique et économie étaient alors étroitement liées. Si ces expéditions ont été bien étudiées par les historiens et que certaines d'entre elles ont marqué l'imaginaire occidental, les expéditions scientifiques contemporaines ont été moins étudiées et sont moins accessibles au grand public. Pourtant, il semble que dans la seconde moitié $d u x^{e}$ siècle et en ce début du $\mathrm{XXI}^{\mathrm{e}}$ siècle, leur nombre soit bien supérieur à celui des expéditions que connut la période coloniale. Comment dès lors interpréter le rôle et la signification des expéditions scientifiques contemporaines qui se déroulent dans des pays indépendants et dans un contexte postcolonial ? Si elles ne participent plus à la construction d'empires coloniaux au sens géographique et géopolitique du terme (il n'est alors en effet plus question de conquête coloniale), continuentelles néanmoins à jouer un rôle politique ? Nous apportent-elles des données ou des points de vue nouveaux sur notre rapport à la science et à la nature?

En partant d'une description et d'une analyse d'une expédition scientifique contemporaine menée sur l'île de Santo au Vanuatu en 2006, je me propose d'apporter dans cet article des éléments de réponse préliminaires à ces questions. Pour ce faire, je resituerai d'abord cette expédition dans le contexte d'une prise de conscience de la crise actuelle de la biodiversité et de l'émergence d'initiatives internationales prises pour y répondre. J'exposerai ensuite les raisons scientifiques et politiques qui ont présidé au choix de l'île de Santo comme site d'inventaire, avant de montrer comment et pourquoi le module ethnologique de l'expédition a été victime du climat international actuel en matière de bioprospection et de biopiraterie. Je poursuivrai par une réflexion sur le rapport à la science et à la nature, en montrant que la privatisation du financement de l'expédition ne signifie pas une marchandisation. Je conclurai sur ce que peut impliquer, pour le rapport occidental à la nature, le succès médiatique d'une expédition ayant accordé une large place aux invertébrés et aux espèces invasives, taxons peu appréciés du " grand public ».
L'expédition Santo 2006 a rassemblé, d'août à décembre 2006, plus de 150 participants (biologistes, ethnologues, plongeurs, spéléologues, journalistes, documentaristes, photographes...) venus d'une vingtaine de pays différents, sans compter les habitants de l'île de Santo qui ont joué un rôle décisif de guides, collecteurs, porteurs, chauffeurs, cuisiniers, étudiants-stagiaires... Le but d'une telle expédition, coorganisée par trois institutions (le Muséum National d'Histoire Naturelle de Paris, MNHN, l'Institut de Recherche pour le Développement, l'IRD, et une ONG, Pro-Natura International), était d'inventorier tous les compartiments de la biodiversité de l'île de Santo et de l'archiver dans les collections des grands muséums d'histoire naturelle. La principale justification avancée par l'initiateur de cette expédition, Philippe Bouchet, biologiste marin et malacologue au MNHN, était la suivante : dans le contexte actuel de crise majeure de la biodiversité, considérée par certains biologistes comme la sixième crise d'extinction des espèces, il faut découvrir et faire l'inventaire de cette biodiversité avant qu'elle ne disparaisse. Pour les organisateurs, l'enjeu d'une telle expédition était donc non seulement d'acquérir de nouveaux savoirs scientifiques sur la biodiversité, avant qu'il ne soit trop tard, mais aussi d'alerter les médias et, par leur intermédiaire, les politiques et l'opinion publique, sur la gravité de la situation et la nécessité de prendre des mesures pour y remédier. L'importance de la couverture médiatique de cette expédition, voulue par les organisateurs, constitue l'une de ses principales originalités. Si de nombreuses expéditions ont pour but d'acquérir des savoirs scientifiques sur certains compartiments de la biodiversité, elles se font habituellement loin des lumières médiatiques, et personne, mise à part la communauté scientifique concernée, n'en est informée. Santo 2006 a donc introduit de ce point de vue une certaine rupture vis-à-vis des autres expéditions scientifiques d'exploration de la biodiversité. Elle se voulut aussi exemplaire en termes d'éthique et de respect des nouvelles règles d'accès à la biodiversité et de partage des avantages découlant de son utilisation définies en 1992 par la Convention sur la diversité biologique (CDB), convention signée par 150 pays dont la France et le Vanuatu ${ }^{2}$. Comme l'a expliqué Philippe Bouchet lors d'une réunion préparatoire à cette expédition en octobre 2005 tenue à l'IRD de Nouméa :

« Le projet Santo est aussi pour moi une occasion d'amener [au Muséum] cette nouvelle culture d'accès à 
la biodiversité qui doit dorénavant passer par des partenariats formels, des volets de formation et des volets de restitution [au profit du pays hôte]. Ce sont les conditions sine qua non pour la durabilité même des institutions comme les musées. " (enregistrement et transcription de la réunion tenue à l'IRD, à Nouméa, en octobre 2005)

Santo 2006 s'inscrit donc bien dans une thématique moderne d'approche scientifique de la biodiversité. Un bref rappel de la conception de cette notion et de sa prise en compte dans nos sociétés occidentales contemporaines nous aidera à nous en convaincre.

\section{Biodiversité et politique}

\section{Le National Forum on Biodiversity de 1986 et le lancement du cri d'alarme sur l'état de la biodiver- sité}

C'est en 1985 que le néologisme biodiversity est créé par Walter G. Rosen lors de la préparation du National Forum on Biological Diversity qui se tiendra l'année suivante à Washington D.C. (Le Guyader 2008). Au cours de ce forum, les biologistes américains font le constat de l'existence d'une crise affectant la biodiversité. D'après de nombreux auteurs, et notamment Virginie Maris (2006), c'est au cours de ce forum que les biologistes lancent le premier grand cri d'alarme concernant la crise de la biodiversité. D'après eux, les espèces vivantes disparaîtraient à un rythme tel que certains n'hésitent pas à parler de sixième extinction, même si une telle expression est controversée par d'autres biologistes (Le Guyader 2008), les cinq autres extinctions, reconnues à l'échelle paléontologique, ayant eu des ampleurs respectives bien supérieures à celle qui touche la biodiversité terrestre aujourd'hui. Pour Virginie Maris (2006) :

« Le constat de l'intérêt croissant de la communauté scientifique pour la question des extinctions d'espèces et la volonté du comité scientifique d'en informer un large public ont convergé vers l'idée d'un forum national sur la diversité biologique. [...] La mission des scientifiques présents était bien davantage d'alerter les autorités politiques et l'opinion publique du danger que représentait la perte de diversité biologique que de décrire le phénomène sous un angle purement scientifique. [...] Ce Forum fut le premier grand plaidoyer en faveur de la conservation de la diversité biologique. »)

Virginie Maris (ibid.) poursuit son analyse en évoquant la conférence de presse au cours de laquelle «ce collectif d'éminents biologistes regroupés sous le nom de Club of Earth annonça que la diversité biologique est dans un état de véritable crise, et que cela représente un grand danger pour l'humanité :

«The species extinction crisis is a threat to civilisation second only to the threat of thermonuclear war. »

Outre le contenu même de cette alerte, l'élément essentiel est, pour Virginie Maris :

« l'ampleur de la couverture médiatique et le relais assuré par les associations environnementales [qui] permirent à l'évènement, auquel assistaient plus de 14000 personnes, de résonner massivement et durablement dans l'espace public. Dès lors, la perception collective de la valeur de la diversité biologique et du danger que représente son érosion ne fera qu'augmenter. Les actes du colloque, rassemblés sous la direction d'Edward O. Wilson dans le désormais célèbre BioDiversity, furent publiés en 1988 à la National Academy Press (Wilson 1988). » (Maris, 2006)

\section{La prise de conscience des années 1980}

Quelques années auparavant, en 1982, Terry Erwin réalise une expérience sur une espèce d'arbre au Panama qu'il pulvérise avec un puissant insecticide (fogging) afin de collecter les insectes présents. Cette expérience va montrer que l'ordre de grandeur de la biodiversité spécifique est beaucoup plus important que ce que l'on soupçonnait jusque-là. Ce constat, inattendu, est édifiant. Pour Le Guyader (2008) :

« Le rêve des naturalistes - décrire exhaustivement l'ensemble des espèces, les répertorier et les classer, s'éloigne. Ensuite, le désaccord sur l'ordre de grandeur (de 10 à 100 millions d'espèces) indique que l'on est incapable de quantifier le nombre d'espèces. Et enfin, étudier la "biodiversité spécifique" nécessite une toute autre force de frappe que celle de quelques naturalistes isolés réalisant, sans trop de moyens, des missions de courte durée dans des environnements riches. »

C'est donc dans les années 1980 que les biologistes découvrent à la fois l'étendue de leur ignorance sur la biodiversité spécifique et les menaces qui pèsent sur elle. Selon Le Guyader (2008), de ces constats découle l'idée qu'il faut dorénavant organiser des expéditions scientifiques de grande taille. Cette conviction va être déterminante pour impulser une série d'initiatives internationales qui, dans les années 1990 et 2000, vont tenter d'apporter des réponses à ces deux problèmes que sont :

- le manque certain de connaissances scientifiques sur la biodiversité ;

- la nécessité et l'urgence d'élaborer des politiques de conservation de la biodiversité avant qu'elle ne disparaisse. 
Le handicap taxonomique et les initiatives internationales pour le surmonter

Or, la discipline qui permet de collecter, identifier et classer des espèces nouvelles pour la science, est la taxonomie. Mais cette discipline est, aux dires de tous, en crise depuis un certain nombre d'années (Tillier, 2000). Ainsi, dès le début des années 1990, les biologistes mentionnent l'existence de ce qu'ils appellent un « handicap taxonomique » qui limite considérablement l'émergence pourtant urgente de nouvelles connaissances sur la biodiversité ainsi que l'élaboration de politiques conservationnistes.

"The taxonomic impediment is a term that describes the gaps of knowledge in our taxonomic system (including knowledge gaps associated with genetic systems), the shortage of trained taxonomists and curators, and the impact these deficiencies have on our ability to manage and use our biological diversity. " (Darwin Declaration, 1998, UNEP/CDB/COP/ /4/Inf.28, 2 may 1998)

Dans les années 1990 et 2000, différentes initiatives internationales, gouvernementales et non gouvernementales, ont tenté de surmonter ce handicap taxonomique et, au-delà, d'apporter des réponses politiques et scientifiques à la crise affectant la biodiversité. Parmi les plus importantes d'entre elles, la signature, en 1992, de la première convention internationale sur la diversité biologique va jouer un rôle déterminant. La CDB va, en effet, modifier les règles du jeu en « nationalisant» la biodiversité qui, de patrimoine commun de l'humanité, devient la propriété d'États souverains. Dès lors, pour avoir accès à la variété des espèces vivantes, que ce soit pour l'étudier scientifiquement ou pour la prospecter dans le but de découvrir de nouvelles substances naturelles commercialisables (pour la pharmacopée, l'agroalimentaire, la cosmétique...), de nouvelles procédures sont nécessaires, comme l'obtention d'un «Consentement Informé Préalable » (CIP) de la part des populations locales concernées et la signature d'une convention avec le pays hôte. De plus, la CDB va introduire la notion de partage des avantages découlant de l'utilisation des ressources génétiques (article 15). La CDB va également lancer, dès 1993, une Initiative Taxonomique Mondiale (Global Taxonomic Initiative), qui sera officiellement mise en œuvre en 2002 et dont le but est de réduire le handicap taxonomique :

"The GTI has been established by the Conference of the Parties (COP) to address the lack of taxonomic information and expertise available in many parts of the world, and thereby to improve decision-making in conservation, sustainable use and equitable sharing of the benefits derived from genetic resources. This is the first time in history that taxonomy has had recognition at such a high level in international policy. » (http://www.cbd.int/gti/)

D'autres initiatives internationales visant à surmonter le handicap taxonomique virent également le jour dans les années $1990^{3}$. Sans en faire une liste exhaustive, on peut citer la création, en 1993, du Réseau Taxonomique Mondial appelé Bionet International qui s'est focalisé sur le renforcement des capacités des pays du sud en matière taxonomique. On peut aussi évoquer la création, en 2002, du Global Biodiversity Information Facility ${ }^{4}$ (GBIF), créé à l'initiative du groupe de travail «Informatique et Biodiversité » de l'ocDE (Organisation de Coopération et de Développement Economique). Le GBIF est un portail internet d'accès aux collections naturalistes numérisées des muséums d'histoire naturelle, herbiers et collections faunistiques. L'objectif de ce portail est de mettre en accès libre et gratuit les informations taxonomiques primaires sur la biodiversité. Cette initiative vise à assurer une meilleure gestion, plus équitable, plus facile d'accès, plus collective des collections existantes sur la biodiversité (http://www.gbif.fr/). Enfin, on peut évoquer la création, en 2002, du Consortium pour le Code barre du Vivant (CBOL) qui promeut une nouvelle technique, moléculaire, d'identification des espèces, censée être beaucoup plus rapide et moins coûteuse que l'identification morphologique (http://www.barcoding. si.edu/). Cette liste, incomplète, révèle l'existence de nouvelles dynamiques, portées par différentes types d'acteurs sociaux, à différentes échelles, visant toutes à surmonter le handicap taxonomique. Leur objectif est de faire de la taxonomie une discipline moderne en utilisant les technologies de la génétique et en mettant à son service des moyens d'information et de communication (informatique et Internet) performants, pour permettre à la taxonomie de relever les nouveaux défis identifiés par les biologistes au milieu des années 1980 :

- accélérer l'acquisition de nouveaux savoirs scientifiques sur la biodiversité

- contribuer à une meilleure gestion et à une conservation efficace de la biodiversité.

Outre de telles initiatives, une réponse d'un autre type est apportée au « handicap taxonomique » : l'élaboration d'un nouveau type d'expé-

3. $C f$. Faugère, Castro, Ollivier (2008) pour une description et une analyse de ces différentes initiatives internationales.

4. Système mondial d'information sur la biodiversité 
ditions naturalistes à l'image de l'expédition Santo 2006.

\section{La genèse d'une expédition : entre science et politique}

De mars 2005 à novembre 2006, j'ai mené des enquêtes ethnographiques ${ }^{5}$ sur la préparation puis le déroulement de cette grande expédition naturaliste. C'est lors d'un entretien que j'ai fait en mars 2005 avec Philippe Bouchet sur le programme de conservation des forêts sèches de Nouvelle-Calédonie (Faugère 2008, ce numéro), que celui-ci m'a proposé de prendre comme objet d'étude l'expédition Santo 2006, qu'il était alors en train d'organiser. L'idée d'impliquer un chercheur en sciences sociales dans l'étude du montage d'une expédition naturaliste lui était venue à la suite de difficultés politiques qu'il avait rencontrées lors de l'organisation d'une précédente expédition, aux Philippines, appelée Panglao 2004. Si l'expédition Santo ressemble aux expéditions antérieures, une analyse plus fine montre qu'elle semble en renouveler le genre. Elle a en effet tenté de " moderniser » la taxonomie en créant une grande expédition pluridisciplinaire d'inventaire de tous les compartiments de la biodiversité - et notamment les plus négligés d'entre elle (invertébrés, champignons, espèces invasives) -, d'y associer des chercheurs en sciences sociales - ethnologues notamment -, de respecter les règles éthiques de la $\mathrm{CDB}$, et d'impliquer des bailleurs de fonds majoritairement privés. L'expédition Santo 2006 et celles qui sont en cours de préparation peuvent être considérées comme des réponses apportées par un collectif de scientifiques à la « crise » actuelle que traverse la connaissance taxonomique de la biodiversité.

C'est le 24 mars 2004 qu'a lieu la première réunion de lancement de ce qui deviendra l'expédition Santo 2006. Le compte rendu de cette réunion, écrit par Philippe Bouchet, ainsi que la déclaration d'intention qu'il rédigera le 4 mai 2004 révèlent que, dès le départ, les principales dimensions de l'expédition étaient présentes. On peut en effet lire que Santo 2006 sera une « opération pluridisciplinaire d'étude de la biodiversité, du patrimoine naturel, et de communication sur cette biodiversité $»$. Dès sa première mouture, le projet Santo propose de fédérer plusieurs types d'expéditions (terrestres, marines et souterraines) qui se déroulent habituellement séparément, d'inclure un volet ethnologique et d'insister sur les obligations éthiques :

«L'opération se veut exemplaire dans le cadre des obligations éthiques et politiques d'un pays du Nord (la France) vis-à-vis d'un pays du Sud, ancienne colonie (le Vanuatu, ancien condominium francobritannique des Nouvelles-Hébrides). Une attention particulière sera portée à la formation et à la restitution de l'information. " (déclaration d'intention, Santo 2006, 4 mai 2004)

Lors de cette première réunion de lancement, l'idée d'y associer l'équipe du «Radeau des Cimes », pour explorer la canopée, est déjà évoquée. Dans cette même déclaration d'intention, le volet ethnologique s'intitule « développement durable et valorisation de la biodiversité », et implique un ethno-pharmacologue de l'IRD de Nouméa et un ethno-écologue du MNHN. Mais ce module sera profondément remanié par la suite en raison de tensions apparues à la fin de l'année 2005 , lors de la signature d'une convention entre les organisateurs de l'expédition et le gouvernement du Vanuatu. J'y reviendrai. La seule composante qui n'était pas encore présente et qui n'apparaîtra qu'un an plus tard, au début de l'année 2005, est celle relative à la biodiversité des espaces anthropisés, incluant un inventaire des espèces végétales et animales invasives (voir l'article Bouchet, Le Guyader et Pascal, dans ce numéro).

Santo 2006 s'inscrit d'abord dans l'histoire des campagnes et ateliers marins organisés dans la région Indo-Pacifique par Philippe Bouchet depuis $1993^{6}$ comme celui-ci l'explique sur le site internet de l'expédition qu'il a organisée en 2004 aux Philippines :

« Les biologistes marins ont reconnu depuis longtemps dans l'océan Pacifique l'existence d'un gradient de biodiversité : les mers d'Asie du Sud-Est consti-

5. Mon matériel d'enquête est composé de l'enregistrement et de la transcription de dix réunions préparatoires qui se sont déroulées d'avril 2005 à juin 2006, de deux réunions postérieures à l'expédition, de sept entretiens réalisés en 2005 avec les organisateurs de l'expédition et avec des membres du service mécénat du Muséum national d'histoire naturelle, de discussions informelles avec les membres du comité directeur de l'expédition et avec différents participants à l'expédition, de la participation à une mission préparatoire de huit jours effectuée au Vanuatu en octobre/novembre 2005 avec Philippe Bouchet et Hervé Le Guyader, de la participation à deux modules de l'expédition elle-même pendant quatre semaines (le module marin et un petit groupe de trois entomologistes et d'une botaniste du module Forêts Montagnes Rivières), de l'accès à l'ensemble des emails que Philippe Bouchet a échangés entre mars 2004 et septembre 2005 au cours du montage de l'expédition, ainsi qu'à de nombreux documents internes (comptes rendus de réunion, versions provisoires des déclarations d'intention, des conventions, etc.).

6. Ses premières campagnes ont commencé en Nouvelle-Calédonie (Koumac, Touho) et ont été les expéditions «fondatrices » même si elles n'avaient pas encore l'ampleur (vingt personnes) de ce qu'elles sont devenues par la suite (Philippe Bouchet, communication personnelle, septembre 2008) 
tuent un "Triangle d'Or" où la richesse spécifique est la plus élevée. Elle s'appauvrit graduellement à mesure que l'on s'éloigne de ce cœur, avec un "pôle froid" en Polynésie orientale. La reconnaissance de ce gradient s'appuie cependant, dans la littérature scientifique, sur un nombre étonnamment faible de données chiffrées, de surcroit très disparates. L'objet du présent projet est précisément de recueillir des données nouvelles sur la richesse spécifique d'un site situé au cœur du "pôle chaud" du gradient de biodiversité marine : Panglao, aux Philippines. Les résultats seront comparés à ceux obtenus à Rapa [en 2002], dans l'archipel des Australes (Polynésie française), qui représente le "pôle froid" de ce gradient, et à Koumac, Touho et Lifou [en 2000] (en Nouvelle-Calédonie), qui occupent une position intermédiaire. » (Philippe Bouchet 2002, http://www. panglao-hotspot.org/Description/francois.html)

Inventorier la biodiversité marine de l'île de Santo, qui se situe, comme la NouvelleCalédonie, dans une position intermédiaire, s'inscrivait donc dans le cadre de ce programme de recherche général sur l'étude du gradient de biodiversité marine dans l'océan Pacifique. L'autre raison scientifique du choix de l'île de Santo est la découverte, quelques années auparavant, de bois coulés au large de Santo. Lors d'une discussion 7 , en octobre 2005, avec Hervé Le Guyader, co-directeur de Santo 2006 pour l'IRD, et professeur de biologie évolutive à l'université Pierre et Marie Curie (Paris 6), celui-ci m'expliqua que les biologistes avaient découvert une forme de vie très particulière se développant au fond des océans autour de bois coulés et de squelettes de baleines. Il poursuivit en disant qu'aux îles Salomon se trouve une grande quantité de bois coulés mais que la situation politique de ce pays y rend les missions scientifiques trop risquées. Ayant découvert en 2004 de nombreux bois coulés autour de l'île de Santo, la décision fut prise d'intégrer leur étude à l'expédition Santo $2006^{8}$.

Des raisons scientifiques et politiques ont donc présidé au choix du Vanuatu et de l'île de Santo. Outre la situation pacifiée de ce pays, d'autres raisons politico-administratives ont également joué un rôle. En 2003 et 2004, lors du montage de l'expédition Panglao aux Philippines, Philippe Bouchet s'était heurté à de nombreuses difficultés administratives et politiques, qui l'avaient conduit à repousser l'expédition d'une année. Au cours d'une réunion prépara- toire qui s'est tenue au MNHN en mai 2005, celui-ci a rappelé :

«La mission au Vanuatu du mois de mars a confirmé que sur le plan politique et diplomatique, cela reste facile de monter des opérations de cette nature dans les petits pays insulaires du Pacifique Sud par rapport aux monstres bureaucratiques et politiques que sont devenus les Philippines, l'Indonésie, le Brésil, l'Inde etc. Au Vanuatu, on a été extrêmement bien reçu tout de suite, et on a pu facilement rencontrer plusieurs ministres. Mais on a pris conscience aussi que s'il était facile d'avoir les bénédictions de nature politique ce n'était pas suffisant. À Santo, le vrai pouvoir d'accueillir et de donner de vraies bénédictions se trouve au niveau des communautés, au niveau de la coutume et des villages. Il est donc essentiel d'avoir une bénédiction au niveau politique parce que c'est avec le gouvernement qu'on signera un Memorandum of Understanding 9 mais cela ne dispense pas d'une très longue phase de préparation sur place, de réunions d'information, de passage dans les villages, etc. » (Philippe Bouchet, réunion mai 2005, enregistrement et transcription par Elsa Faugère)

Si les démarches semblaient facilitées par la petite taille du pays qui permettait un accès rapide et facile aux plus hautes sphères de l'État, et par la dimension restreinte de l'administration qui limitait les procédures bureaucratiques, la signature de la convention entre le consortium des trois organisateurs (MNHN, Pro-Natura International, IRD) et le gouvernement du Vanuatu révèlera finalement un certain nombre de difficultés identiques à celles que Philippe Bouchet avaient rencontrées aux Philippines. Ce sont ces tensions et ces difficultés qui vont conduire à une modification en profondeur du volet ethnologique de l'expédition.

\section{«Savoirs locaux » et bioprospection : les aléas du module ethnologique}

C'est le $1^{\mathrm{er}}$ novembre 2005 , lors d'une réunion à Port Vila à laquelle j'ai assisté aux côtés de deux organisateurs de l'expédition, Hervé Le Guyader (IRD) et Philippe Bouchet (MNHN) et de Dona Katafalk, membre du service de l'environnement du gouvernement du Vanuatu, que certaines tensions ont surgi ${ }^{10}$. L'objet de cette réunion était de discuter de la première version du Memorandum of Understanding, envoyée quel-

7. Cahier de terrain Elsa Faugère 2005.

8. Une mission spécifique, appelée SantoBoa, centrée sur les bois coulés et organisée par Bertrand Richer de Forges (IRD) et Sarah Samadi (IRD), a été réalisée dans le cadre de l'expédition Santo 2006.

9. Convention signée entre les organisateurs de l'expédition et le gouvernement du Vanuatu.

10. J'ai retracé en détail cette entrevue dans le séminaire des sociologues de l'INRA de juin 2006 ( $c f$. Faugère 2006), ainsi que dans un article en collaboration avec Sélim Louafi et soumis à la revue Genèses le 31 mars 2008, intitulé « La nature, la science, l'État et l'indigène. Le cas de l'Expédition Santo 2006 ». 
ques jours auparavant par les organisateurs au service de l'environnement du gouvernement du Vanuatu. Alors que nous avions rencontré cette personne quelques jours plus tôt dans une atmosphère cordiale, ce jour-là, l'ambiance fut glaciale. La principale pomme de discorde a porté, de manière inattendue pour moi, sur le module ethnologique de l'expédition.

Comme je l'ai déjà mentionné, l'expédition comportait, dans la première déclaration d'intention, un module intitulé « développement durable et valorisation de la biodiversité » avec des retombées prévues en termes d'écotourisme, mais dont le contenu exact n'était pas encore précisé. Ce n'est qu'au début de l'année 2005 que le contenu de ce module deviendra plus précis, s'intitulant alors «Diversité culturelle, perceptions et usages ». Il s'agissait de faire une comparaison des inventaires « savants» et «populaires" de la diversité biologique dans le domaine de la phyto-pharmacologie traditionnelle et des plantes tinctoriales. Pour ce faire, les ethno-écologues de ce module allaient devoir recueillir « des savoirs locaux sur la biodiversité » (Expédition Santo 2006, document provisoire du 20 février 2005). Cet objectif restera inchangé jusqu'à la fin de $2005^{11}$. Et c'est précisément cet aspect qui a posé problème à Dona Katafalk et à d'autres membres du service de l'environnement. Ceux-ci semblaient en effet craindre que les ethno-écologues de l'expédition utilisent des " savoirs locaux » des habitants de Santo à des fins commerciales, en l'absence de tout contrôle de leur part, sans leur verser de contreparties et sans avoir préalablement obtenu leur consentement informé.

Début décembre 2005, Russel Nari, alors directeur du service de l'Environnement du Vanuatu, envoie à Philippe Bouchet une nouvelle version de la convention. Les modifications apportées montrent clairement que le gouvernement du Vanuatu veut encadrer strictement les recherches menées dans le module ethnologique sur les savoirs locaux pour s'assurer de bien en contrôler les procédures et les utilisations en cas de bioprospection. Le premier ajout concerne un préambule qui stipule que les bailleurs de fonds doivent être mentionnés afin de s'assurer que si une industrie pharmaceutique ou appartenant à tout autre secteur commercial est impliquée, une convention spécifique soit alors signée, en plus de celle-ci. Le deuxième porte sur un long paragraphe ajouté dans l'article 2 (les obligations des organisateurs de l'expédition (MOU, 2005) qui détaille la procédure à suivre pour collecter des «savoirs locaux» sur la biodiversité, pour les utiliser et pour les conserver. Deux éléments apparaissent essentiels : la consultation du Centre Culturel du Vanuatu à toutes les étapes de la procédure (pour la collecte, la publication et l'archivage), et l'obtention du Consentement Informé Préalable de la part des habitants de Santo concernés. Si les organisateurs avaient bien mentionné, dans la première version de la convention, l'obligation d'obtenir le Consentement Informé Préalable de la part des habitants de Santo avant d'effectuer toute collecte naturaliste, ils n'avaient pas précisé que cela concernait également le recueil des " savoirs locaux » sur la biodiversité. De plus, ils n'avaient pas mentionné le Centre culturel du Vanuatu ignorant alors le rôle-clé que celui-ci jouait dans l'accès aux savoirs locaux, dans leur utilisation et leur conservation $^{12}$. Enfin, dans l'article 4, sont apportées des précisions concernant les questions de propriété intellectuelle sur les « savoirs traditionnels et indigènes $»$.

L'ensemble de ces remarques, ajouts et discussions a finalement conduit les organisateurs à supprimer le volet ethno-pharmacologique et toute autre question portant sur les usages locaux de la biodiversité afin de n'être pas soupçonnés de biopiraterie. Ce soupçon aurait en effet pu mettre en péril l'ensemble de l'expédition dont l'objectif principal était de réaliser un inventaire scientifique de la biodiversité (et non un inventaire des savoirs locaux sur la biodiversité). Dans un courriel envoyé le 22 décembre 2005 à Pierre Cabalion, l'ethno-pharmacologue de l'expédition, Philippe Bouchet lui expose très clairement les raisons de ce qu'il intitule «le recentrage du module ethnosciences » :

\section{«Salut Pierre,}

Lors de notre passage au Vanuatu, j'avais commencé à sentir arriver les problèmes autour du projet de convention (MOU) dont j'avais discuté avec toi et que j'avais soumis à Russel [Nari]. Ces craintes ont été confirmées lors du passage d'Olivier Pascal et de Bruno Corbara qui a suivi le nôtre [...]. J'ai eu moimême ces dernières semaines des échanges de mails avec Russel, d'où il ressort que c'est un peu l'ensemble

11. La seule modification apportée dans ce module au cours de l'année 2005 sera la mention de ma participation qui figure, dans le document officiel daté de septembre 2005, de la manière suivante : «Enfin, SANTO 2006 est en lui-même un sujet d'étude socio-ethnologique sur les motivations économiques, politiques, éthiques et intellectuelles des acteurs du projet» (Expédition Santo 2006, document du 15 septembre 2005).

12. Sur l'histoire de la politique culturelle du Vanuatu, $c f$. Bolton (1999) et Tryon (1999). Voir également le document « Réglementation de la recherche culturelle au Vanuatu », http://www.vanuatuculture.org/research/20070108_reglementation_ de_la_recherche_culturelle.shtml. 
du module "Ethnosciences" qui fait difficulté, et en particulier un blocage net sur tout ce qui touche aux "usages". Tu avais trouvé que mon projet de MOU n'allait pas assez loin pour les objectifs qui t'intéressent; le gouvernement du Vanuatu, lui, trouve que notre proposition va déjà beaucoup trop loin. Je joins à ce mail les ajouts et corrections au MOU qui m'ont été adressées par Russell : tu constateras que "l'encadrement" des ethnosciences est devenu extrêmement étroit. Il est clair que derrière "ethnopharmacologie", ils ont vu molécules et plus, et du coup la suspicion s'est abattue sur tout Santo 2006. Nous avons discuté au Comité directeur des conséquences de ce durcissement/de cette méfiance des autorités du Vanuatu à l'égard de notre projet. Nous ne voulons pas passer pour des biopirates, et nous ne voulons pas que l'objectif général d'inventaire soit jugé à travers le prisme de l'ethnopharmacologie et des "plantes utiles". Nous voulons envoyer un signal fort qu'on a écouté ce qu'ils nous ont dit, et supprimer en conséquence de notre programme scientifique les recherches correspondant à la phrase : A current research theme on many continents is a comparison between "academic" and "popular" biodiversity inventories of vertebrates and plants. In Santo, this comparison will address indigeneous phytopharmacology and economic botany. "

Pourtant, si le gouvernement du Vanuatu voulait clairement encadrer et structurer les modalités de collecte et d'utilisation des « savoirs traditionnels et indigènes ", il ne demandait pas aux organisateurs de supprimer toute recherche sur ce thème. Je pense, au contraire, que c'était un sujet qui intéressait au premier plan le gouvernement en raison de ses éventuelles retombées commerciales. D'ailleurs, à la même période, le gouvernement du Vanuatu autorisait une biologiste allemande à venir travailler sur les connaissances médicinales « traditionnelles » des habitants du pays. Ses recherches consistaient explicitement à tester les usages des plantes traditionnelles en termes de propriétés médicinales et pharmaceutiques. Il s'agissait bien, pour elle, de se baser sur les savoirs médicinaux traditionnels pour tenter de repérer des propriétés médicales et pharmaceutiques de ces plantes. L'objectif de ses recherches était donc clairement de la bioprospection. Le gouvernement du Vanuatu n'était pas hostile à la bioprospection en soi. On peut même dire qu'au contraire, la possibilité de bénéficier de retombées économiques et financières des recherches scientifiques l'intéressait au plus haut point. Mais ce qui l'inquiétait dans l'expédition Santo c'était de ne pas pouvoir contrôler les éventuelles retombées commerciales, étant donnés l'ampleur et la taille de la mission, et les dizaines de milliers d'échantillons qui allaient être récoltés par les scientifiques. À mes yeux, le problème semblait être plutôt celui du contrôle que celui de la nature de l'activité menée.
Compte tenu du climat international très tendu sur ces questions de bioprospection et de biopiraterie, les organisateurs de Santo ont préféré ne pas prendre de risques et ne pas mettre en péril le cœur de l'expédition qui était d'inventorier et d'archiver la biodiversité de cette île. Ils étaient avant tout dans une logique de production de connaissances académiques sur la nature, même s'ils étaient ouverts, au départ, aux aspects de valorisation économique de la biodiversité qui, dans la mesure où elle bénéficie de manière équitable au pays fournisseur de la biodiversité et aux chercheurs, est pleinement dans l'esprit de la CDB.

En dépit de la prudence des organisateurs de Santo 2006 qui les avait conduits à supprimer tout aspect pouvant être taxé de bioprospection, un article est paru le 28 juin 2006 dans le Daily Post (Willie, 2006), le seul quotidien du Vanuatu. Dans cet article, le journaliste reprend les propos $\mathrm{du}$ directeur du Centre Culturel du Vanuatu, Ralph Regenvanu, qui met en garde son gouvernement contre les dangers de biopiraterie liés à l'expédition Santo 2006, comme, dit-il, à toute recherche scientifique naturaliste menée par des organismes étrangers au Vanuatu. Considérant que son pays ne dispose pas encore des moyens pour contrôler efficacement de telles recherches, il propose d'y interdire toute recherche naturaliste, sur le modèle du moratoire de dix ans qui avait interdit, de 1984 à 1995, les recherches en sciences humaines au Vanuatu. Pour appuyer sa démonstration, il prend l'exemple de brevets déposés par des chercheurs étrangers sur le kava, à partir des connaissances « traditionnelles » des populations locales et dont ces dernières ne tirent aucun bénéfice. Et il insiste sur le potentiel très lucratif des recherches scientifiques menées sur la biodiversité, dont les populations locales peuvent attendre d'énormes bénéfices financiers.

Cet article sera sans conséquence sur la mise en œuvre de l'expédition Santo 2006, la convention ayant déjà été signée avec le gouvernement. Le directeur du Centre culturel voulait, semblet-il, profiter de ce contexte particulier pour faire passer ce message auprès de son gouvernement et le pousser à créer un Conseil national scientifique et un Conseil de la Biodiversité qui serait chargé de mieux contrôler les recherches biologiques étrangères et de mieux en négocier les contreparties.

La question des contreparties est au cœur des critiques émises par Ralph Regenvanu (voir l'article de Fabienne Tzerikiantz, dans ce numéro) qui considère que les rapports et les productions académiques ne sont d'aucune utilité pour les habitants de la côte ouest de Santo. 
Ce point de vue n'était pas partagé par tous les membres du gouvernement du Vanuatu. Lors d'un entretien qu'Hervé Le Guyader et moi avons eu, le 3 novembre 2005, avec Russel Nari, alors directeur du service de l'environnement, celui-ci a clairement manifesté son enthousiasme pour l'expédition Santo, considérant qu'elle serait d'une grande utilité pour la politique environnementale de son pays. À ses yeux, les résultats de l'expédition Santo 2006 allaient aider le gouvernement à redéfinir une politique de protection de la biodiversité solidement ancrée dans des faits scientifiques.

Cette controverse met en lumière l'existence de deux postures divergentes dans le rapport à la nature et à la science. Il semble y avoir d'un côté les tenants d'un rapport marchand à la science et à la nature qui plaident en faveur de recherches à finalités socioéconomiques, seules à même de délivrer des contreparties qu'ils considèrent comme acceptables, et de l'autre, les tenants d'un rapport non marchand à la science et à la nature pour lesquels l'acquisition de savoirs académiques sur la biodiversité constitue un objectif légitime en soi, même si ces savoirs ne débouchent sur aucun usage économique commercialisable.

De manière a priori paradoxale, les tenants du rapport «non marchand» à la science et à la nature, comme les organisateurs de l'expédition Santo 2006, étaient pourtant majoritairement financés par des fondations d'entreprises privées (Fondation Niarchos, Fondation Total...). On aurait pu s'attendre à ce que la privatisation du financement de l'expédition conduise à une marchandisation. Or, cela n'a pas été le cas.

\section{Mécènes et sponsors, le soutien du privé}

L'expédition Santo a été majoritairement financée par du mécénat de fondations d'entreprises. Pour Philippe Bouchet, il était clair qu'une expédition de cette ampleur et de cette nature ne pouvait pas être entièrement financée par de l'argent public et qu'elle devait trouver des soutiens privés. Dans un entretien effectué en mai 2005, Philippe Bouchet raconte que l'idée de prospecter auprès des fondations privées lui était venue au début des années 1980 à la suite d'un séjour aux États-Unis où il avait pu constater dans des laboratoires de recherche la diversité des sources de financement émanant, notamment, des fondations d'entreprises. À son retour en France, il a contacté les quelques fondations d'entreprises qui existaient alors. Mais ses demandes furent rejetées au motif que celles-ci ne finançaient que de la recherche dite fonda- mentale, ce qui signifiait alors pour elles, de la recherche biomédicale.

En juillet 1999, alors qu'il était en train de préparer un atelier marin à Lifou (en NouvelleCalédonie), il prit contact avec la Fondation Total pour la Biodiversité et la Mer, sur les conseils d'un de ses collègues du British Museum. Il leur présenta alors le projet Lifou. Lorsqu'il reçut une réponse de leur part début 2000, il s'était passé deux événements majeurs entretemps : la fusion Total-Fina et l'affaire de l'Erika. Ces deux événements avaient conduit Total à augmenter le budget de la Fondation, tout en conservant le même nombre de projets soutenus. Philippe Bouchet s'est donc - involontairement - rapproché de la Fondation à un moment favorable. La Fondation accepta alors de financer l'expédition marine de Lifou. Philippe Bouchet raconte dans cet entretien :

« L'expédition à Lifou a été super vis-à-vis de Total en termes de retour d'images. On a eu une bonne couverture médias. Ils étaient très contents de Lifou parce que, malgré tout, le Muséum est encore un endroit prestigieux. » (Entretien avec Philippe Bouchet, 4 mai 2005, enregistrement et transcription par Elsa Faugère)

Depuis lors, toutes les expéditions montées par Philippe Bouchet (à Rapa en 2002, à Panglao en 2004 et à Santo en 2006) ont reçu un soutien financier de la fondation Total. Ces soutiens financiers, sous forme de mécénat, laissent une totale liberté d'action aux scientifiques. Les mécènes attendent un retour d'image ce qui implique une bonne couverture médiatique. De plus, Philippe Bouchet est régulièrement sollicité par la fondation Total pour faire des conférences sur la biodiversité - auprès du Club d'actionnaires de Total, auprès d'un groupe biodiversité existant au sein du groupe Total et auprès du World Environment Center, club de multinationales (de l'automobile, de l'agroalimentaire, de la chimie, du pétrole, des mines, des communications) qui affichent des préoccupations pour l'environnement et le développement durable.

« Avec la fondation Total, on a un peu une relation, je dirai, d'obligations réciproques. Je trouve ça intéressant de faire ces conférences. Je n'ai absolument pas l'impression, j'allais dire, de me faire acheter. La société dans laquelle on vit n'est pas faite que d'organismes de recherche et de crédits de recherche publique. Et ça me sert, je pense, à être plus convaincant quand je vends mes projets scientifiques à un ministre du Vanuatu ou à un directeur d'organismes aux Philippines. Je ne parle pas qu'un langage académicoscientifique. Avec ce genre de contacts, je comprends mieux quel regard les autres peuvent porter sur mon 
activité professionnelle et sur la biodiversité. » (Entretien avec Philippe Bouchet, 4 mai 2005, enregistrement et transcription par Elsa Faugère)

La privatisation du financement de l'expédition Santo n'a attiré ni polémique ni critique sur la qualité scientifique des recherches menées, leur intégrité et leur indépendance. L'ensemble des participants à cette expédition, ethnologues inclus, a accepté d'être financé par des fondations d'entreprises.

En septembre 2005, alors même que le budget de l'expédition était loin d'être bouclé, puisque seulement $10 \%$ avaient alors été trouvés, les organisateurs ont refusé une importante proposition financière émanant d'une entreprise fabriquant des parfums et des arômes alimentaires. Pour fabriquer ces produits, l'entreprise travaille sur des substances naturelles et fait donc de la bioprospection. Pour les organisateurs, le dilemme fut alors le suivant : peut-on accepter de l'argent d'une entreprise qui fait de la bioprospection, même si leur aide, dans le cadre de l'expédition Santo, n'est que du mécénat? Il était d'emblée clair pour ces organisateurs que si cette entreprise voulait participer financièrement à l'expédition Santo dans le but de faire de la bioprospection, la réponse devait être négative, en raison, notamment, du contexte international actuel sur ces questions hautement sensibles déjà évoqué. Mais s'il ne s'agissait que de mécénat, pourquoi refuser une aide dont ils avaient alors cruellement besoin? Aux yeux des organisateurs, il était préférable de refuser cette subvention même s'il ne s'agissait que de mécénat, afin de ne pas risquer de porter préjudice, en termes d'images, à l'ensemble de l'expédition.

La logique du mécénat est celle du don. C'est une transaction monétaire mais non marchande ${ }^{13}$. Sans développer ici une analyse anthropologique du don, on voit, par cet exemple, que certains dons sont refusés car ils sont considérés comme risqués, comme si le don portait avec lui quelque chose de l'identité de son donateur, comme s'il allait "coller à la peau » des donataires ainsi que l'exprime l'un des organisateurs :

«Est-ce que l'on a envie de se coller avec cette entreprise ? On peut se demander si à long terme c'est très profitable d'avoir ce genre d'étiquette qui te colle à la peau. » (Extrait d'une réunion préparatoire de l'expédition Santo, 15 septembre 2005, enregistrement et transcription par Elsa Faugère)
Que des fondations d'entreprise aient accepté de financer, sous forme de mécénat, une expédition comme Santo 2006, révèle plusieurs tendances de fond de l'économie capitaliste contemporaine :

- d'une part, un renouveau de la philanthropie et du mécénat depuis le début des années 1980, processus que certains sociologues, comme Nicolas Guilhot (2004), lient à la financiarisation de l'économie capitaliste depuis cette période ;

- d'autre part, l'apparition et le développement d'entreprises qui se disent socialement et écologiquement responsables.

Pour l'ethnologue, ces liens entre financements privés, exploration scientifique et conservation de la biodiversité constituent une piste d'investigation particulièrement novatrice et intéressante à approfondir. Des questions émergent en effet sur la signification et les conséquences de ce mouvement de privatisation de certains volets de la recherche académique. Si, dans ce cas précis, il n'y a pas eu de marchandisation des résultats de l'expédition et si les scientifiques ont bénéficié d'une totale marge de manœuvre, ce mouvement semble révéler un désengagement de l'État qui mériterait des réflexions et des enquêtes approfondies.

\section{L'attention portée aux invertébrés et aux espèces invasives}

Je voudrais terminer cet article par ce qui a fait le cœur de l'expédition Santo 2006 : l'inventaire des compartiments, négligés, de la biodiversité, c'est-à-dire des invertébrés (terrestres et marins), des champignons mais aussi des espèces végétales et animales invasives. Cette focalisation sur la biodiversité oubliée, qui ne fait généralement pas la une des médias ni même n'intéresse les politiques de conservation, constitue un des grands défis qu'ont tenté de relever ces naturalistes et ce type d'expédition. D'ailleurs, le succès de Santo 2006, en termes scientifiques et médiatiques, a conduit les organisateurs à mettre en œuvre un nouveau plan stratégique de 10 ans " pour compléter la connaissance sur la biodiversité des 11 points chauds de la planète" (http://www. pronatura.org/projects/planet_reviewed_fr.pdf). Ce plan, intitulé « La planète revisitée. Vers un renouveau des grandes expéditions naturalis-

13. Un certain nombre de travaux anthropologiques et sociologiques ont analysé les distinctions entre transactions marchandes et non marchandes, monétaires et non monétaires ainsi que les significations sous-jacentes de l'argent que ces distinctions révèlent $-c f$. notamment Zelizer (1994), Weber (2000) et Faugère (2000). 
tes », consiste en l'organisation de grandes expéditions qui se focalisent sur certains compartiments négligés de la biodiversité (invertébrés et champignons) http://www.pronatura.org/ projects/planet_reviewed_fr.pdf). En déplaçant le regard sur ces taxons et en s'en faisant les porte-parole, les naturalistes leur donnent une existence sociale, amplifiée par une forte couverture médiatique qui modifiera peut-être le rapport occidental à la nature.

\section{En guise de conclusion}

Les jeux politiques et géopolitiques dans lesquels l'expédition Santo a été prise, les controverses et les tensions qu'elle a provoquées révèlent un changement dans les rapports entre pays $\mathrm{du}$ sud, fournisseurs de biodiversité, et pays du nord, demandeurs d'accès à celle-ci. En cherchant dès le départ à inscrire l'expédition dans le cadre éthique fixée par la CDB, en pensant développer un aspect de valorisation économique et en associant des sciences humaines, les organisateurs ont tenté de créer un nouveau style d'expédition naturaliste, tenant mieux compte de certaines dynamiques contemporaines.

\section{Remerciements}

Je remercie Philippe Bouchet de m'avoir donné la chance d'ethnographier l'expédition Santo, en me témoignant une grande confiance tout au long de ce travail. Je remercie aussi les deux autres organisateurs, Olivier Pascal et Hervé Le Guyader, ainsi que l'ensemble du comité scientifique de l'expédition et tous les participants (scientifiques et non scientifiques) qui ont toujours accepté avec bienveillance et curiosité ma présence et mes questions. Il va sans dire que sans leur aide et leur collaboration précieuse, ce travail n'aurait pas pu exister. Mes enquêtes au Vanuatu ont été financées par le budget de l'expédition Santo 2006.

\section{BIBLIOGRAPHIE}

Bolton Lissant, 1999. Introduction, Oceania 70, 1, pp. 1-8.

Consortium POUR LE CODE BARRE DU VIVANT, http://www.barcoding.si.edu.
DARWIn DeClaration, 1998. UNEP/CBD/COP/4/Inf.28, 2 may 1998, http://www.cbd.int/doc/meetings/cop/ cop-04/information/cop-04-inf-28-en.pdf.

FAugère Elsa, Monica CASTro et Guillaume OlliVIER, 2008. Taxonomy's rebirth triggered by the international biodiversity policies: An historical and anthropological approach, in $14^{e}$ International Symposium on Society and Resource Management, Burlington, Etats-Unis.

FAUGÈRE Elsa, 2000. Transactions monétaires en pays kanak, Genèses 42, pp. 41-62.

—, 2006 (juin). Anthropologie de la biodiversité : l'expédition Santo 2006. Premiers éléments, communication à la $\mathrm{VII}^{\mathrm{e}}$ Journée des sociologues de l'INRA.

GLOBAL BIODIVERSITY INFORMATION FACILITY. http://www.gbif.fr.

Global TAXonomy Initiative. http://www.cbd.int/ gti.

GuIlHot Nicolas, 2004, Financiers, philanthropes. Vocations éthiques et reproduction du capital à Wall Street depuis 1970, Paris, éditions Raisons d'Agir.

LE Guyader Hervé, 2008. La biodiversité : un concept flou ou une réalité scientifique ?, Courrier de l'environnement de l'INRA 55, pp. 7-26.

MARIS Virginie, 2006. La protection de la biodiversité : entre science, éthique et politique, thèse de philosophie, université de Montréal.

RÉglementation DE LA RECherche CUltuRELLE AU VANUATU,

http://www.vanuatuculture.org/research/20070108_ reglementation_de_la_recherche_culturelle.shtml.

RoYSON Willie, 2006 (june 28). Scientific research gets firts butting from govt institution, Daily Post 1764.

Site OFFICIEl DE L'EXPÉDition Panglao, http://www. panglao-hotspot.org/Description/francois.html.

Site OFFICIEL DE PRONATURA INTERNATIONAL, http:// www.pronatura.org/projects/planet_reviewed_fr.pdf.

Tillier Simon (éd.), 2000. Systématique. Ordonner la diversité du vivant, «livre blanc », Éditions Tec \& Doc.

TrYON Darell, 1999. Ni-Vanuatu Research and Researchers, Oceania 70, 1, pp. 9-15.

WEBER Florence, 2000. Transactions marchandes, échanges rituels, relations personnelles. Une ethnographie économique après le grand partage, Genèses 41, pp. 85-107.

ZeLIZER Viviana, 1994. The social meaning of money, New York, Basic Books. 
